\title{
Daylighting Strategy for Sustainable Schools: Case Study of Prototype Classrooms in Libya
}

\author{
Belal Abdelatia (Corresponding author) \\ GRECAU Laboratory \\ Higher National School of Architecture and Landscape Architecture of Bordeaux \\ 70109 - 33405 Talence cedex, France \\ Tel: 33-05-5735-1163 E-mail: belal.abdelatia@bordeaux.archi.fr \\ Christian Marenne \\ CERMA Laboratory Architectural and Urban Ambient Environment \\ 16202 - 44262 Nantes Cedex2, France \\ Tel: 33-02-4059-1719 E-mail: christian.marenne@cerma.archi.fr \\ Catherine Semidor \\ GRECAU Laboratory \\ Higher National School of Architecture and Landscape Architecture of Bordeaux \\ 70109 - 33405 Talence cedex, France \\ Tel: 33 (0)5 57351159 E-mail: catherine.semidor@bordeaux.archi.fr
}

\begin{abstract}
Over the last few years, topics related to sustainable development and energy saving in Western countries led to several research projects aiming to promote the amount of natural daylight used in school buildings. The same issues arise now in Libya, especially those concerning the use of natural daylight. The promotion of natural daylight in the classrooms saves electrical energy and improves the working conditions for both teachers and students. The extreme solar heat in Libya has always been an imperative factor in relation to building heat. Especially during the period from June to August great care is given to avoid overheating while still preserving the use of natural daylight.

The present research aims to propose and to test several architectural devices that can be integrated into educational building designs, allowing the penetration of natural daylight while respecting the traditional aestheticism and creating a comfortable school environment.
\end{abstract}

Keywords: Classrooms, Natural daylight, Visual comfort, Sustainable visual environments, Sustainability

\section{Introduction}

Student's learning capacity and performance, such as the ability to concentrate during the lessons, do not only depend on individual characteristics, such as motivation, psychological conditions, intelligence, etc. but also on several other external factors that affect not only the student, but the overall environment of the school.

Visual comfort is one of the main features that contribute to the creation of a suitable educational environment. This comfort is defined as "a subjective feeling of satisfaction with the visual system mainly due to the absence of any discomfort induced by the overall visual environment" (AFE, 1995).

Therefore, a person should feel comfortable in an environment where the lighting is not too bright and illuminates properly the worktables and spaces necessary for the daily activities.

Because visual activities such as reading and writing are very important during the educational phase, it is essential to create comfortable visual conditions in school buildings that will contribute to these activities.

Furthermore, the use of natural daylight in buildings significantly reduces the electric energy consumption, especially in the secondary and tertiary sectors (Page, J. et al, 1994). For instance, it has been shown that artificial lighting of non-domestic buildings represents $50 \%$ of the energy consumption in Europe (Scartezzini, 1991). It also has been shown that it is possible to reduce this consumption with 30 to 70 percent by combining the use of artificial and natural lighting (Mc. Nicholl, A. and Owen L. J, 1994).

Internationally, these studies aroused a growing interest in natural daylight as an efficient way to save and rationalize the energy consumption in buildings. Indeed, today the use of natural daylight is considered as one of the main strategies for all bioclimatic, passive solar and/or high quality environmental design.

According to Veitch ( 2004), a Canadian researcher specialized in the interaction between the quality and usage of light, there is no real consensus on what good quality lighting means. On the contrary, concerning artificial lighting, it is generally accepted that the lighting, luminance, luminance repartition (contrast between the surfaces), uniformity, glare control, flickering of fluorescent light tubes and the spectral power distribution, are 
all important aspects of a luminous environment.

Veitch proposes to deepen the concept of quality by the study of the interaction between the inhabitants and the environment. She observes that the evaluation of the quality of a natural lighted space involves the consideration of a multitude of factors (Veitch, 2004). She also proposes an integrated model of the light quality as illustrated in figure 1 . Thus, this quality model acknowledges that the response of the occupier to his/her light environment is contextual. That it depends on his or her personal characteristics, the layout of his or her environment, and the culture. Moreover, according to this model, the well-being of the inhabitant (see upper part of the diagram) does not only depend on the notion of visibility, but also on different factors specific to the photobiology.

Research also confirmed that the visual comfort related to the quantitative and qualitative aspects of the natural daylight significantly contributes to the well-being of pupils and thus leads to better school performances. A research under more than 20,000 elementary students and 100 schools in the USA confirmed this statement. It has been proved that students with the most natural lighting in their classroom progressed $26 \%$ faster in reading and 20\% faster in mathematics (Heschong Mahone Group, 1999). The present research has been motivated by this specific link between an improvement of the light quality and school performance increase.

Although the natural light in Libya, due to an always clear sky, is potentially sufficient to supply the necessary lighting of its buildings, and the resort to permanent artificial light limited, a protection against the hot solar radiation is in return necessary. It is this issue which the author wishes to address in this study of strategies for natural classroom lighting in hot and dry climates.

\section{Sustainable Visual Environments}

The idea of sustainability is an important part of contemporary architecture. Concerns related to the creation of sustainable architecture, sometimes wrongly narrowed down to "low energy building" concepts, in fact go far beyond the energy related issues. While they are focused on environmental targets, they must also take into account factors such as the building's aesthetics, functions, internal microclimate and other aspects which affect the human's psychophysical state.

Two elements of sustainable building design which have direct effects on student performance are natural daylighting and indoor air quality. Studies now show that better indoor air quality in schools results in healthier students and faculty, which in turn results in fewer student absences and further improves student achievement (Karanikoloudis G. 2005). Recent studies on the effect of natural daylight in schools reveal besides different health benefits, that students perform better in daylit classrooms.

The presence of daylight in buildings maximizes architectural potential while increasing human comfort and visual perception. Architecture literally depends on light, be it natural or artificial. As light reveals the forms and the spaces created within a building, it simultaneously reveals the design and intention for which it was originally designed and extends its value beyond mere functional use (Guzowsky, 2000).

To guarantee a comfortable and 'healthy' luminous environment, and control the amount and distribution of natural light that enters a space, a good daylighting strategy is essential. This is generally composed of more than a simple opening in the façade (window) or on the roof (skylight). Depending on the local climate, and the building's orientation, functions and requirements, customized solutions or devices may need to be implemented.

Daylighting systems can range from simple static features (louvers, light-shelves, fixed overhangs, laser-cut panels, prismatic elements, anidolic systems, etc.) to adaptable dynamic elements (blinds, movable lamellae, advanced glazing, holographic optical elements, etc.) and/or a combination of these elements (Tuaycharoen et al., 2007). The palette of devices available is very broad. Numerous cutting-edge techniques are constantly being implemented in the field of design to increase daylight penetration within indoor spaces, improve distribution and uniformity, control direct sunlight and/or reduce glare (Wienold et al., 2006). It should be noted, however, that although numerous methods have been developed for the assessment and prevention of glare from natural light, no method is currently available to assess the simultaneous impact of both daylight and artificial lighting sources on the perception of glare (Boyce et al., 2003).

Any given visual activity has different relationships with the spaces surrounding it. The architectural spaces must meet very complex requirements, including the various human needs for a specific orientation in space and time and also needs to be related to the society and culture. Indeed, light, both natural and artificial, plays a key-role in creating a mood and an atmosphere that should meet an occupants' expectations (functions, aesthetics, ergonomics, etc.) and demands (privacy, concentration, etc.), while facilitating perception and expressing a design message of its own (Kramer, 2001).

Thus daylight, in the context of design, should not be considered as an afterthought, taken into account after the spatial characters of the building have already taken shape. Rather, daylight should be valued as a necessity that literally drives and directs the design of a built environment. From its early stages of conception and development, daylight should dictate the quality of the internal spaces, ultimately leading to buildings which are economically cheaper to run, less harmful for the environment, and, above all, healthy, inspiring and stimulating for their occupants. 


\section{Methodology}

Because of its very high sunshine levels, Libya's architecture is particular attentive to the protection of school buildings and the window openings against solar radiation. This helps reducing the excess heat caused by the sun, as well as the glare caused by direct sun radiation and the extreme luminous differentiation in indoor spaces. With respect to the classroom's orientation, the solar protection is usually obtained through wall ledges or semi transparent curtains.

The consideration of natural lighting in the research phases is a main objective. Few studies consider the impact of solar protections on the natural lighting. In certain cases, artificial lighting is even used because the solar protections are too efficient. This thus leads to an over consumption of energy while the solar protection is meant to reduce the energy costs. Thus, it is important to find a compromise between efficient solar protection and suitable natural lighting inside the buildings.

In sub-tropical climates, as in Libya, the main sources of energy consumption inside school buildings are the lighting and air conditioning. Between these two sources, lighting represents $80 \%$ of the total energy consumption. Moreover, the fact to use the air conditioning to compensate the heat of the light represents a significant part of the total cooling costs of a building during the summer months. This is why many researchers actively explore the possibilities to incorporate daylight in the architectural lighting design, in order to reduce the energy costs of the buildings (M. Bodart, A. De Herde, 1987).

In order to do this, the research team studied the traditional orientations of school buildings. For example, school buildings with openings on the South and East façades are more protected from the uncomfortable dominant winds. The team elaborated natural light strategies according to their uses and temporality.

Initially, a simulation method will be validated, which will be used to evaluate the proposals which will be presented in the continuation of this thesis, and which will be the result of the inventory of the methods which are found in the traditional way of building in Libya. The classroom which was studied here to develop the work method is a standard, recently built class room, with openings missing sun protection on one side.

\section{Natural lighting strategies}

Natural lighting strategies aim to capture and spread a maximum of natural daylight to avoid visual discomfort. A clever use of natural daylight will thus help reducing the energy consumption spent on lighting while still providing adequate illumination.

These natural lighting strategies must be designed to simultaneously meet the needs of the users and the requirements of the building, thus finding a balance between the conflicting needs of transmission and protection. Specifying daylighting solutions for energy efficiency, comfort and well-being can, however, be a very complex task. They are often highly dependent on climate, latitude, orientation and function, in which many factors and variables can diverge from each other thus making the selection and optimization of different daylighting systems extremely difficult.

This study focused on the following strategies for daylight usage:

- Spreading out of natural daylight in such a way that it creates a good distribution of the light inside the building and facilitates its penetration in a given space.

- Protection against solar radiation or glaring light which have negative consequences on the spatial activities, such as reflected sunshine glaring on the desks, chalkboards and walls.

- Control of the natural daylight in order to manage the quantity and distribution of light in a given space according to the needs of its occupants.

\section{Combined lighting}

A class room cannot be adequately lit by natural light all year long. It has to be combined with artificial light for night-time use and as an additional source of light during days when natural daylight is insufficient. Artificial and natural lighting must therefore be considered from a complementary point of view and not separately. Figures 2 and 3 explain the mechanisms of energy saving strategies through the use of natural daylight. It also illustrates the variations of natural and artificial lighting for a classroom whose dimensions are $6 \mathrm{~m}$ long and $3 \mathrm{~m}$ high.

This combination of artificial and natural lighting results in a significant saving of electrical energy, while providing a good level of visual comfort in indoor spaces. For that purpose, it is necessary to know how much artificial daylight is required. This will automatically send us back to the availability of natural daylight, its daily and seasonal variations, and its penetration in indoor spaces.

\section{Classroom's specifications}

The classrooms selected for this study are selected from the Primary School Prototype Projects (see Figure. 4), and have little controlled natural lighting. The classroom is usually occupied from 7:00 a.m to 3:00 p.m , and is side-lit with unilateral windows that account for $20 \%$ of the floor area. 
The school is located in Ghadames, latitude $30.1^{\circ} \mathrm{N}$, longitude $9.5^{\circ} \mathrm{E}$, with a desert climate. The classroom is on the ground floor of a building facing east and south. The dimensions of the classroom are $6 \mathrm{~m}$ wide $\mathrm{x} 8 \mathrm{~m}$ long $\mathrm{x}$ $3.18 \mathrm{~m}$ high. The working plane is situated at a height of $0.85 \mathrm{~m}$ above floor level.

The finishing materials of the classroom were selected among the materials that are generally used in primary school contruction:

- Floor: stone coverings, reflection coefficient 25 to $45 \%$

- Walls: cream paint reflection coefficient 60 to $70 \%$

- Ceiling: white paint, reflection coefficient 70 to $80 \%$

- Ground coverings: concrete, reflection coefficient 20 to $30 \%$

It is assumed that there is no building in the vicinity that could obstruct direct light from entering the windows. This information will be used for the computer simulation.

\section{Artificial lighting system}

The following is a description of the Artificial Lightig system used in Primary school Buildings.

Even though primary school buildings are mainly used during the day, an artificial lighting system may have to be used in case of insufficient daylight. In the prototype projects, the classroom is fitted with six lights, each using two $58 \mathrm{~W}$ tubular fluorescent lamps. The lights are fitted on two rows perpendicular to the windows. Both rows are connected to one single switch (see Figure. 5).

Schools are usually not equipped with an automatic lighting control system or a control according the availability of natural daylight. Obviously, a substantial amount of energy could be saved if the use of light was more strictly adapted to the occupation level and use of the class rooms (Rea, M.S., Dillon, R.F. et Levy, A.W, 1987).

\section{Prediction and assessment method}

The objective of a method designed to assess the lighting level within a certain space is to determine the quantity and distribution of the penetrating natural daylight (Konya, A, 1984). Under clear skies, the quantity and quality of the light are continuously changing. Indeed, within less than 12 hours, a space is often lit by several sources (sun, sky vault, reflected light, etc.) with sunbeams coming from different directions (see Figure. 6).

In desert areas, the direct light radiation is combined with the reflected radiation. The sky can be described as clear, blue and cloudless. Thus, in such a physical environment, the direct sources of natural daylight present a tremendous importance.

It is thus crucial to stop direct sunshine penetrating in the classrooms during class hours, generally, between 8:00 and 3:00 p.m. Protections designed on the specific dates of 22 June and 22 December (summer and winter solstices) during the above-mentioned hours, will be efficient all year-round (see Figure. 7).

\subsection{Daylight Factor (DF)}

For the evaluation of the day-lighting conditions prevailing in classrooms, where the adequacy of daylight is the main objective, the daylight factor was considered as the most appropriate parameter for indicating the quantity of admitted daylight and consequently the efficiency of the day-lighting design. The daylight factor values (CIE overcast sky) were calculated using DIAL-EUROPE software (see Figure. 8).

One notes through Figure 8 that the percentage of the DF near the windows and in the middle of the classroom exceeds the reference value of $2 \%$, which can create situations of backlighting, strong glare and of overheating due to the penetration of the sun in the classroom.

\subsection{Scale model simulation}

Scale model simulation is a technique used to assess natural day-lighting linked to sunshine in indoor spaces. This simulation method, still widely recognized today, is nevertheless an old method and has been used since 1920 (Willbold-Lohr, G, 1988). The scale model simulation for the evaluation of the lighting does not pose a problem because the length of visible light wave is very short compared to the dimensions of the object to be studied. The scaling down of the simulated item will only slightly modify this relationship and does not affect the internal or external lighting conditions (Papamichael, K. et Beltran, L, 1993).

The scale model was designed on the basis of a typical classroom. The use of a scale model was first preferred to digital simulation because of the sensitive aspect of the atmosphere images that they produce, reconstituting a better feeling of indoor atmospheres. The model allows identifying the secondary effects of sunlight penetration in the classrooms, such as the glare induced by solar stains on the desks, chalkboards and walls (see Figure. 9).

One notes through Figure 9 that the East oriented classroom benefits from the morning sun but the solar radiation is then difficult to control because the sun is low on the horizon. During the winter months, the sunlight is not as strong but penetrates farther into the classroom than in summertime when the sun is high in the sky. On the other hand, in summer, the eastern orientation presents a higher solar exposure than the southern orientation, therefore, there is a real risk of glare and the sun can induce a significant overheating, which is not in the 
student's best interest.

\section{Conclusion}

From these models, one can note that the studied classroom poses problems in terms of luminous comfort and overheating necessitating a reconstruction of the design of teaching spaces while better controlling the luminous qualities of the spaces. These initial results induce the hope to explore other solutions that will result from the study of the architectural devices implemented in the traditional Libyan style of building and to research how they can be used in a modern context with newly evolved construction methods and materials.

This study will provide appropriate daylight strategies for achieving the quality of light needed to improve student's academic performances as well as saving electrical funds.

In conclusion, this study presents a serious endeavor to apply sustainable architectural design strategies to utilize natural daylight. With an ever-growing energy demand, rapidly developing countries such as Libya require ecological building strategies that will improve the illumination in classroom-like environments. This equilibrium is thus obtained while still achieving important human satisfaction and productivity.

The concept of sustainability has to go beyond the exclusive optimization of ranging energy performance factors which lead to a reduction of consumption and environmental impacts. It must also acknowledge the physical, physiological and psychological human needs. Sustainability calls for long-term changes through the interplay of several interconnected systems, adopting an integrated approach to architecture where a decision in one area can influence another. In this context, daylight is an intriguing aspect of design in which environmental, energetic, aesthetic, social, cultural, financial and human aspects simultaneously work together.

Thus, this first study makes it possible to develop various evaluation methods that are simple to implement and provide easily workable results for the natural lighting of classrooms from a quantitative and qualitative point of view.

\section{References}

Association Française de l'Éclairage (AFE). (1995). Vocabulaire de l'éclairage, Paris : Société d'Éditions Lux. Boyce, P., Hunter, C., Howlett, O. (2003). The benefits of daylight through windows. Troy (NY): Lighting Research Center.

Guzowski, M. (2000). Daylighting for sustainable design. New York: Mc Graw-Hill.

Heschong Mahone Group. (1999). Daylighting in schools: An investigation into the relationship between daylighting and student performance. Report submitted in The Pacific Gas and Electric Company on behalf of the California Board for Energy Efficiency Third Party Program.

Karanikoloudis G. (2005). Study of design strategies for the improvement of daylighting in a typical classroom. Diploma thesis submitted in the Department of Civil Engineering, Aristotle University of Thessaloniki.

Konya, A. (1984). Design Primer for Hot Climates. Architectural Press, London.

Kramer, H. (2001). Mastering office lighting. International Lighting Review, Philips Lighting.

M. Bodart, A. De Herde. (1987). Global energy savings in offices buildings by the use of daylighting, Energy and Buildings 34.

Mc. Nicholl, A. and Owen L. J. (Eds). (1994). Daylighting in Buildings. Maxi-Brochure prepare par Energy Research Group University College Dublin pour European Commission DG XVII for Energy.

Page, J et al. (1994). Daylighting developments .A European Perspective. Proceedings European Directory on Energy Efficient Buildings.

Papamichael, K. et Beltran, L. (1993). Simulating the daylight perfomance of fenestration systems and spaces of arbitrary complexity: The IDC method. Proc. of 3rd Int. Conf. Building Simulation'93, 16-18/08/93, Adelaide, Australia.

Rea, M.S., Dillon, R.F. et Levy, A.W. (1987). The effectiveness of light switch reminders in reducing light usage. Lighting Research and Technology.

Scartezzini. (1991). L'éclairage naturel dans le bâtiment. Proceedings CISBAT'91, 10-11/10/91, EPFL, Lausanne.

Tuaycharoen, N., Tregenza, P.R. (2007). View and discomfort glare from windows. Lighting Research and Technology, 39.

Veitch, J. A. (2004). What's new in lighting research? The broad view, Proceedings of International Symposium on Workplace Lighting, Dublin, Ireland.

Wienold, J., Christoffersen, J. (2006). Evaluation methods and development of a new glare prediction model for daylight environments with the use of CCD cameras. Energy and Buildings, 38.

Willbold-Lohr, G. (1988). Scale model as design tool. Proc. Of Building 2000, Barcelona. 


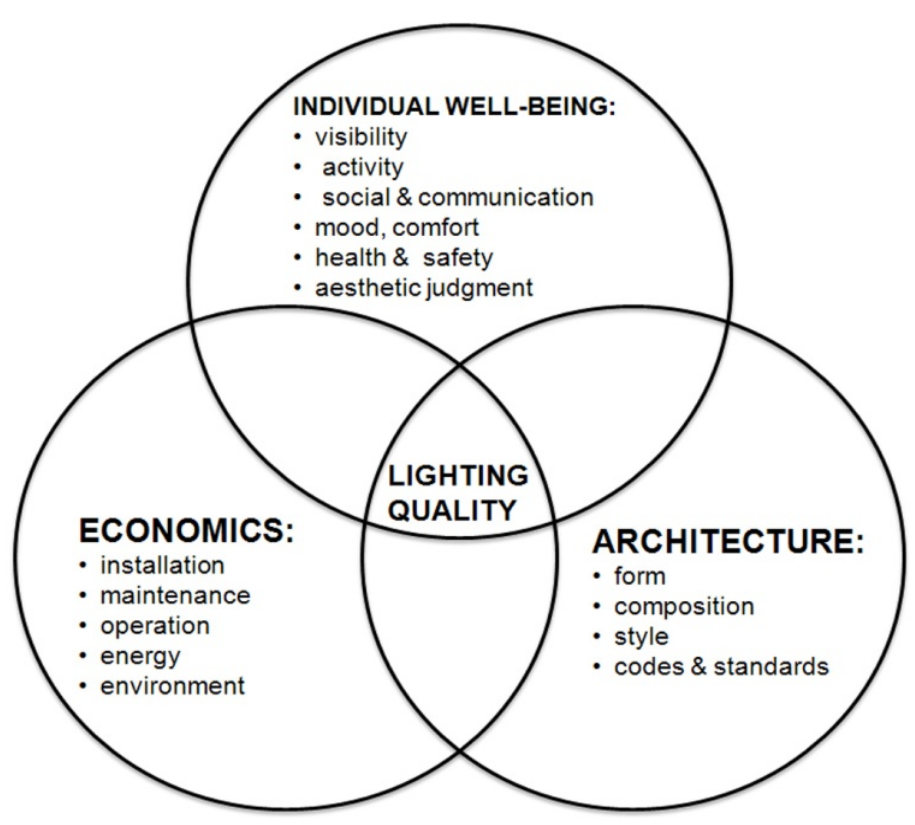

Figure 1. Integrated model of the light quality (source: Veitch, 2004).

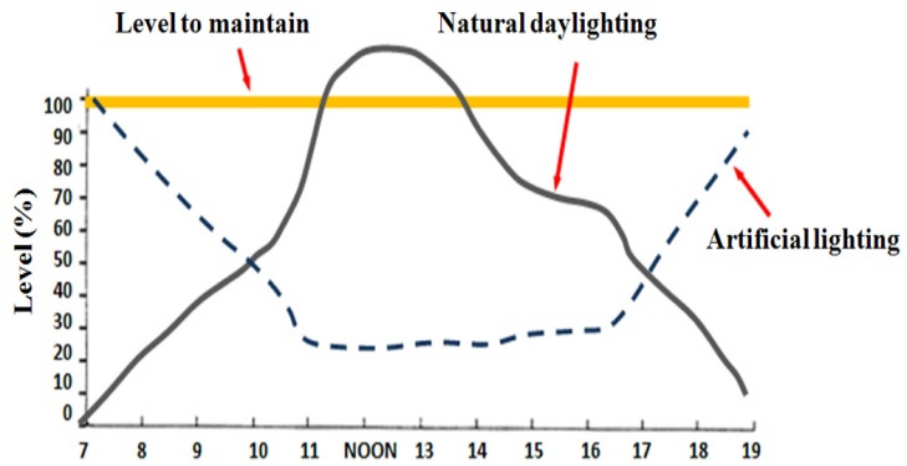

Figure 2. Principles of the combined lighting strategy: to increase the illumination level by using artificial lighting in places where the natural daylight is not enough.

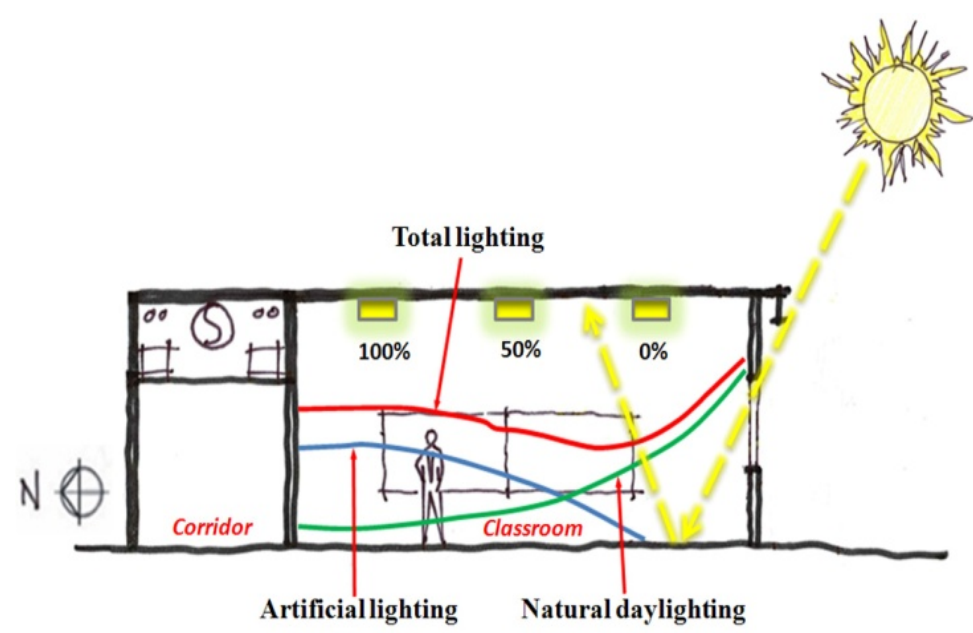

Figure 3. The combined lighting strategy (source: Drawing made by author). 

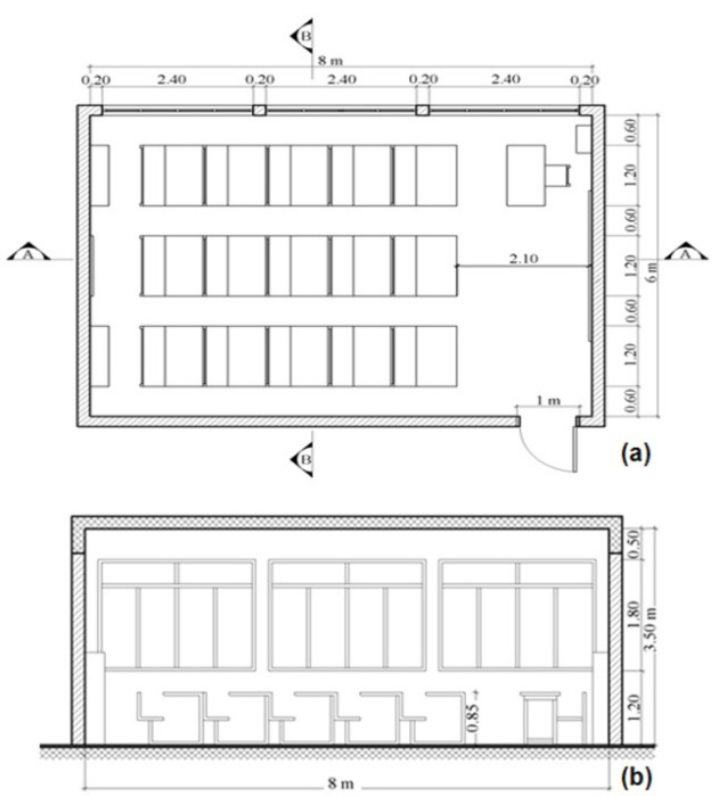

Figure 4. Plan (a), section (b) of a typical classroom in Libya (source: Drawing made by author).
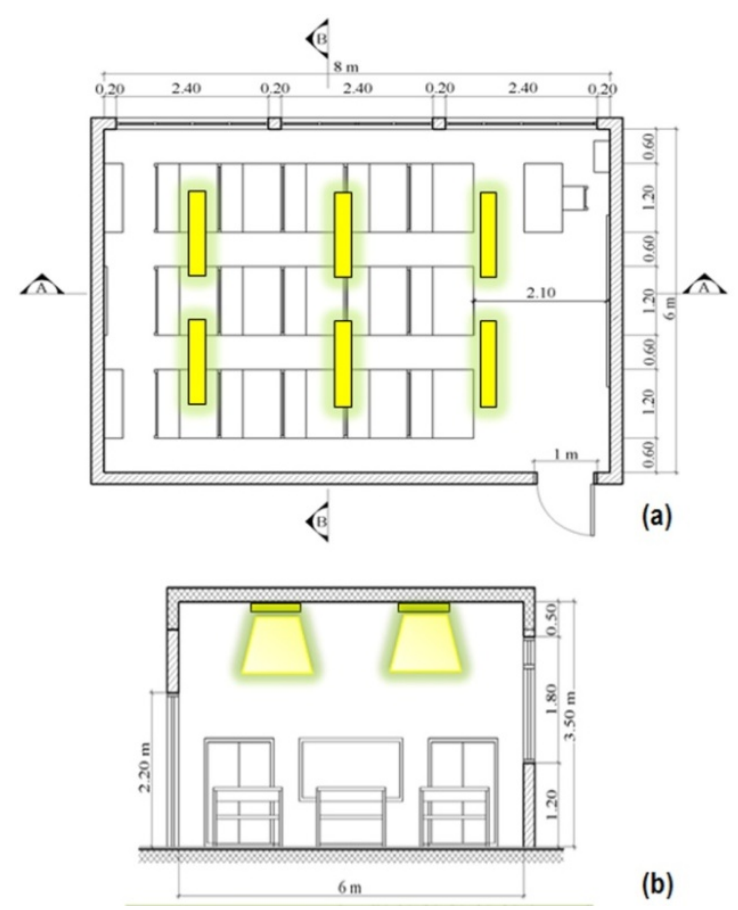

(b)

Figure 5. Plan (a) and section (b) of the artificial lighting system of a typical classroom in Libya (source: Drawing made by author).

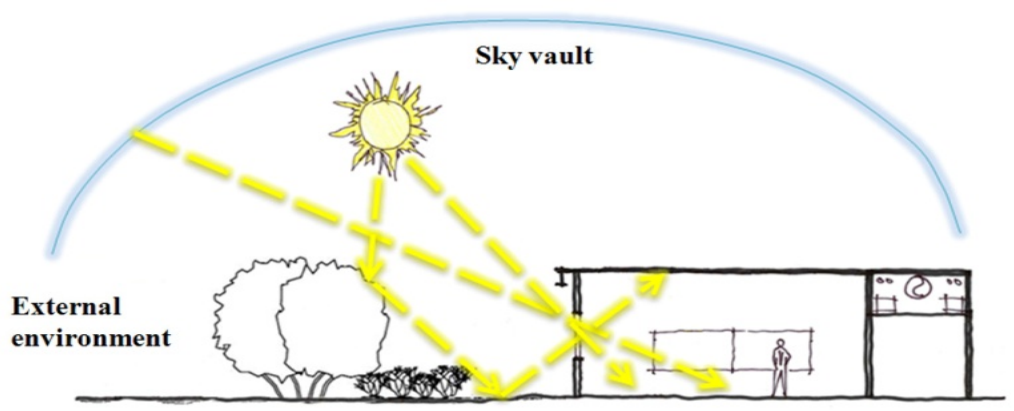

Figure 6. The different external daylighting sources in a building. 


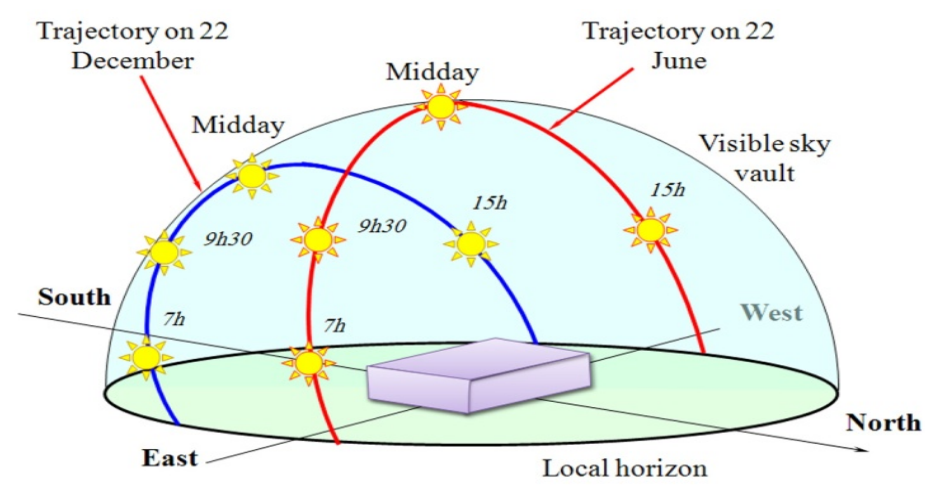

Figure 7. The visible movement of the sun.

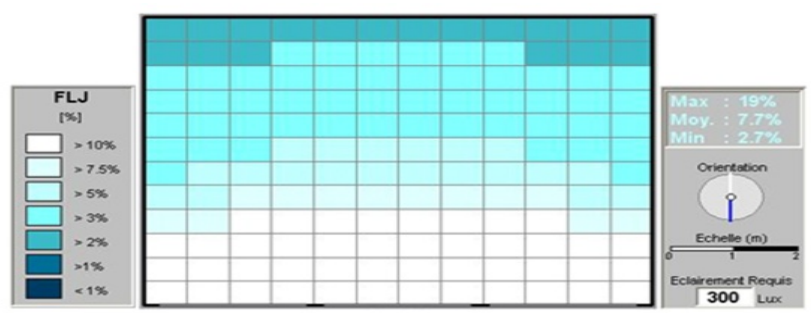

(a)

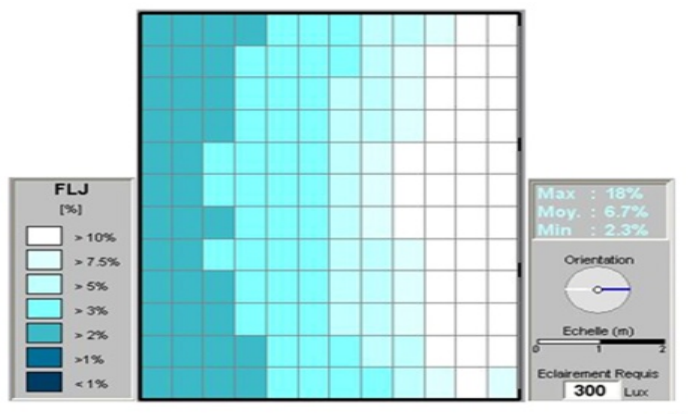

(b)

Figure 8. Distribution of daylight in a South (a) and East (b) oriented classroom.

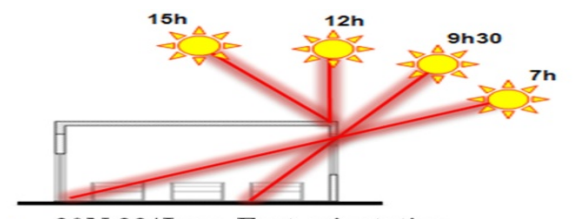

30 N.22/June, East orientation.

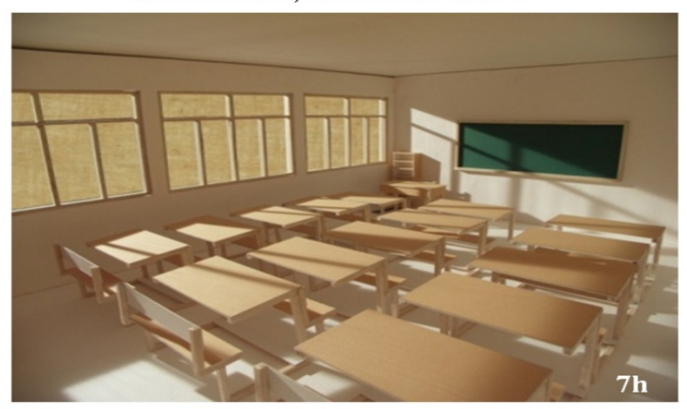

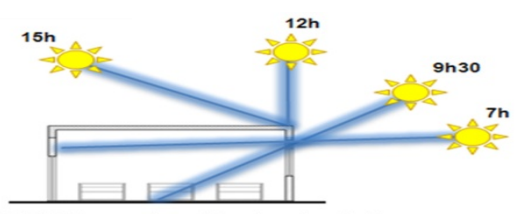

30N.22/December, East orientation.

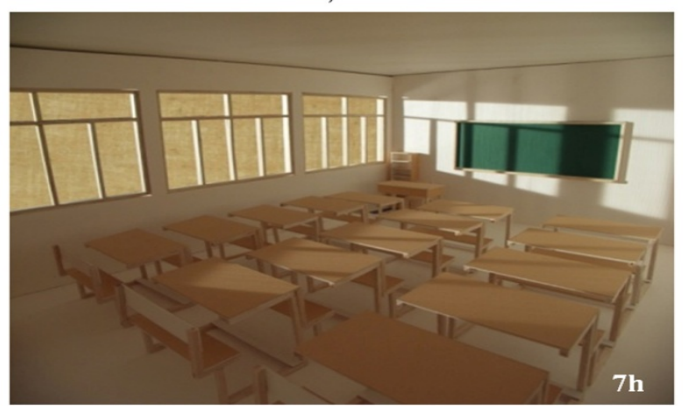

Figure 9. Use of scale models for day-lighting simulation (diagram of the penetration of direct sunlight in a classroom, $30^{\circ} \mathrm{N}$ latitude), (source: Drawing made by author). 